\title{
Identifizierung einer bisher nicht zugeordneten Pollenform in mittelamerikanischen Honigen (sog 'Cuba-Form'): Aeschynomene americana L (Leguminosae, Papilionoideae)
}

\author{
K von der Ohe, JH Dustmann \\ Niedersächsisches Landesinstitut für Bienenkunde, Wehlstraße 4a, D-29221 Celle, Deutschland
}

(Eingegangen 3 April 1996; angenommen 14 May 1996)

\begin{abstract}
Zusammenfassung - Seit Jahrzehnten wird eine bisher nicht identifizierte Pollenform in mittelamerikanischen Honigen als sog 'Cuba-Form' bezeichnet. Im Rahmen einer palynologischen Bearbeitung von Pollenproben aus Äthiopien konnte der Pollen dank charakteristischer Merkmale Aeschynomene americana $L$ (Leguminosae, Papilionoideae) zugeordnet werden. Es handelt sich hierbei um eine Pflanze, die im tropischen und subtropischen Amerika beheimatet ist. Die Klima- und Vegetationsverhältnisse bei der Verbreitung unterschiedlicher Aeschynomene-Arten sowie Beschreibungen zur Herkunft, Häufigkeit und Bedeutung von Ae americana als Bienen Trachtpflanze bestätigen das Ergebnis.
\end{abstract}

Melissopalynologie / Mittelamerika / Cuba-Form

\section{EINLEITUNG}

Honig aus dem mittelamerikanischen Raum wird in erheblichem Maß nach Europa exportiert und liefert einen äußerst wichtigen Anteil für den gesamten Honigkonsum. Vor einer Vermarktung werden diese Honige auf ihre geographische und botanische Herkunft untersucht, abhängig davon, ob der Honig unter entsprechenden Angaben in den Handel gelangen soll. Die deutsche Honig-VO schreibt bei botanischen Angaben ein Überwiegen, bei geographischen
Angaben Ausschließlichkeit der angegeben Tracht vor. Zu diesem Zweck werden melissopalynologische Analysen vorgenommen. Ferner kann anhand der Pollenanalyse im Rahmen der Marktkontrolle das Verschneiden von Honigen unterschiedlicher Provenienzen aufgedeckt werden.

Seit Jahrzehnten wird eine bisher nicht identifizierte Pollenform, die besonders in Honigen aus Cuba in Erscheinung trat, unter den Melissopalynologen als sog 'CubaForm' bezeichnet. Dieser Pollen wurde ausschließlich in Honigen aus weiteren mittel- 
amerikanischen Provenienzen - wie zum Beispiel aus Yucatan und Chiapas in Mexico oder Guatemala (Henrich, mündliche Mitteilung) - nachgewiesen und ist daher als sicherer Anzeiger für diese geographische Region anzusehen (Abb 1). Da die 'CubaForm' durchaus in einer Häufigkeit von bis zu $16 \%$ im Honig festgestellt werden kann, ist davon auszugehen, daß es sich hierbei um eine für Bienen attraktive, eventuell in größerem Umfang vorkommende Trachtpflanze handelt. Aus den oben genannten Gründen besteht seit langem ein dringendes Interesse, das Geheimnis der 'CubaForm' zu enträtseln - nicht zuletzt aus dem Grund, diese Pollen als das Produkt nektarspendender oder nektarloser Trachtpflanzen einstufen zu können. Dies ist von besonderer Bedeutung bei der Bewertung eines botanisch gekennzeichneten Honigs mit Hilfe des Kriteriums der Pollenanalyse.

Im Rahmen der palynologischen Bearbeitung der Pollensammlung zu dem Buch über äthiopische Bienen-Trachtpflanzen (Fichtl und Adi, 1994) konnte der bisher nicht zuzuordnende Pollen dank besonders charakteristischer Merkmale identifiziert werden.

\section{MATERIAL UND METHODE}

Von dem aus Äthiopien stammenden, im National Herbarium of the Addis Ababa University botanisch bestimmten Pflanzenmaterial (Fichtl und Adi, 1994) von Aeschynomene americana wurden gemäß den "Methods of Melissopalynology" (Louveaux et al, 1963) Pollen-Vergleichspräparate für die Lichtmikroskopie (LM) in Glycerin-Gelatine angefertigt. Hierbei wurde auf die Acetolyse verzichtet, da weitergehende Untersuchungen mit dem Raster-Elektronenmikroskop (REM) erfolgten. Die Präparation und Aufbereitung der Pollen für die REM-Analyse wurde nach Von der Ohe und Dustmann (1990) durchgeführt. Zusätzliche vergleichende lichtmikroskopische Honigpräparate sowie authentische Honigproben aus Cuba und Mexico zur weiteren REM-Analyse erhielten wir von W Henrich (Dibona Markenvertrieb KG, Bargteheide). Von diesen Honigproben wurden nach oben angegebenen Methoden ebenfalls LM- und REM-Präparate angefertigt.
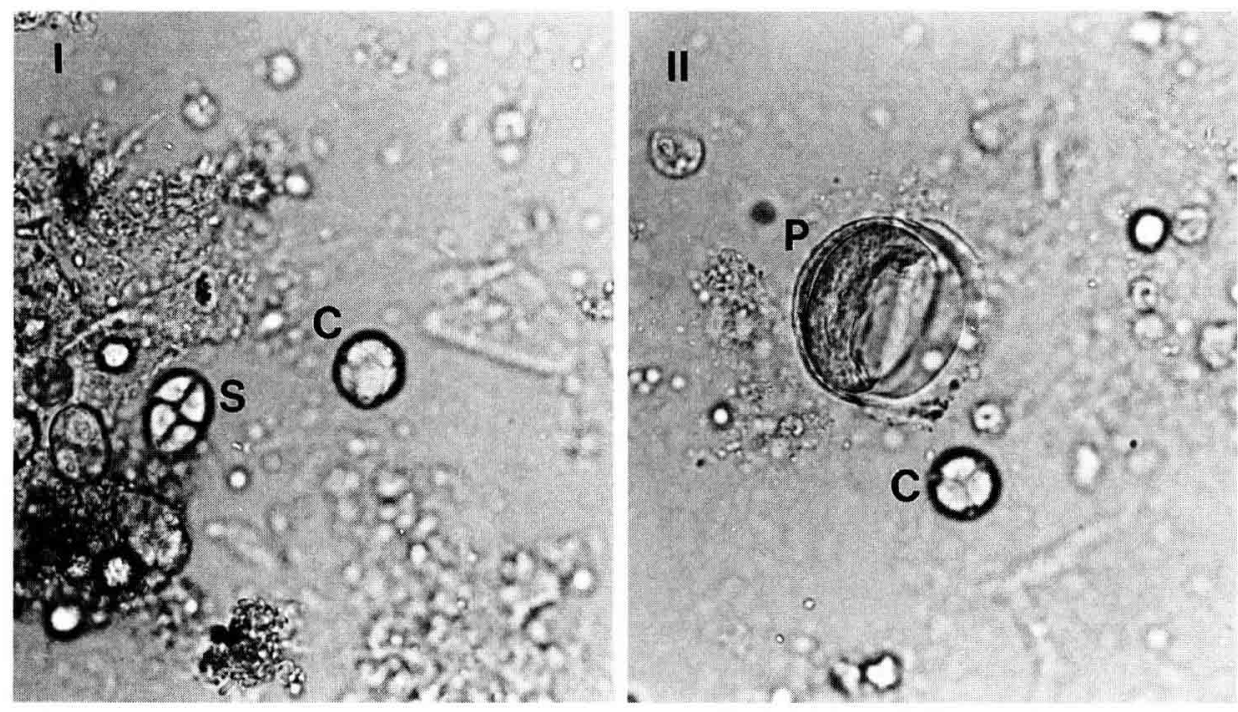

Abb 1. LM-Aufnahmen: Cuba-Form (C) in Mexico (I)- und Cuba (II)-Honig in Begleitung von Palmae (P)- bzw. Schrankia (S)-Pollen, x 475. 
Lichtmikroskopische Aufnahmen wurden mit dem Zeiss Photomikroskop III sowie dem Olympus $A X 70$ erstellt, die REM-Arbeiten wurden am ISI-Super-II durchgeführt.

Die pollenmorphologischen Beschreibungen erfolgten nach der Terminologie von Erdtman (1952) und Faegri (1993).

\section{ERGEBNISSE UND DISKUSSION}

Zur Feststellung der Pollenmorphologie von Aeschynomene americana sowie der 'CubaForm' wurden folgende Parameter herangezogen:

Form; Länge der Polachse und der Äquatorialachse ( $P \times E)$; Anzahl, Art und Anordnung der Aperturen; Exine-Struktur und Skulptur; Farbe.

In allen Kriterien wiesen die 'Cuba-Formen' aus dem mexikanischen bzw cubanischen Honig und der Pollen von Aeschynomene americana identische Merkmale auf (Abb 2 und 3), so daß die folgenden pollenmorphologischen Beschreibungen für alle 3 untersuchten Pollenformen gelten:

Form: inter-semiangular bis circular, 3-colporat, $\mathrm{P} 16 \mu \mathrm{m}(15-17 \mu \mathrm{m}) \times \mathrm{E} 18,5 \mu \mathrm{m}$ $(17-20 \mu \mathrm{m})$.

Aperturen: syncolporat, ora lolongat (endexinöser longitudinaler colpus), colpi mit operculum, margo im Äquatorbereich psilat.

Exine: Sexine reticulat, heterobrochat, lumina bis zu $0,5 \mu \mathrm{m}$, muri simplicolumellat.

Farbe: farblos, grau.

Die deutlichen Übereinstimmungen in der Morphologie lassen den Schluß zu, daß es sich bei der in mittelamerikanischen Honigen vorkommenden 'Cuba-Form' um den Pollen von Aeschynomene americana handelt. Es muß hierbei jedoch bedacht werden, daß ein gleiches Aussehen von Pollen anderer Arten dieser Gattung nicht von vornherein ausgeschlossen werden kann.
Aeschynomene ist sowohl in der palynologischen als auch in der botanischen Literatur nur relativ selten beschrieben worden. In einer Arbeit über südafrikanische Pollen (van Zinderen Bakker, 1959) wurde die Gattung Aeschynomene mit den Arten Ae elaphroxylon und $A e$ nyassana vorgestellt. Die übergeordnete Beschreibung der Gattung schließt die der hier erfassten Art ein. Ae elaphroxylon weicht jedoch in der Größe, Ae nyassana geringfügig in der Gestalt der endexinösen Apertur ab, wobei sich die Angaben auf acetolysierte Pollen beziehen. Die Verbreitung ist mit "kosmopolitisch" (am häufigsten in Afrika) angegeben. Willis (1973) beschreibt die Verbreitung der Gattung Aeschynomene mit "tropisch und subtropisch".

Im nördlichen Australien, der North Kimberley Region, wurde das Vorkommen von Aeschynomene indica beschrieben (Beard, 1990). Bei diesem Gebiet in der Northern Province handelt es sich um ein high-grass savanna woodland, dessen Klima als heißtropisch angegeben ist. Das Kraut Ae indica wächst dort neben Gräsern unter anderem in Gemeinschaft mit Acacia-, Bauhinia- und Eucalyptus-Arten. In der Vegetationsmonographie über Australien von Beadle (1981) wird Ae indica als ein Mitglied einer niedrigen Leguminosen-Gemeinschaft in der bluegrass-savanne der feuchtesten nördlichen Areale erwähnt. Die Honigproduktion Australiens findet vornehmlich im Osten bzw. Südwesten des Kontinents statt. Dies erklärt, warum Aeschynomene-Pollen in australischen Honigen bisher nicht hervorgetreten sind. Nach Zander (1993) ist Ae indica ursprünglich in Indien beheimatet.

Die Gebiete, aus denen vornehmlich die mittelamerikanischen Honige stammen (Cuba, Mexico, Guatemala, El Salvador) sind gekennzeichnet durch Feuchtwälder mit starken Trockenperioden bei großer Verbreitung von Feuchtsavannen sowie regengrüne Trockenwälder, gegenwärtig in großer 

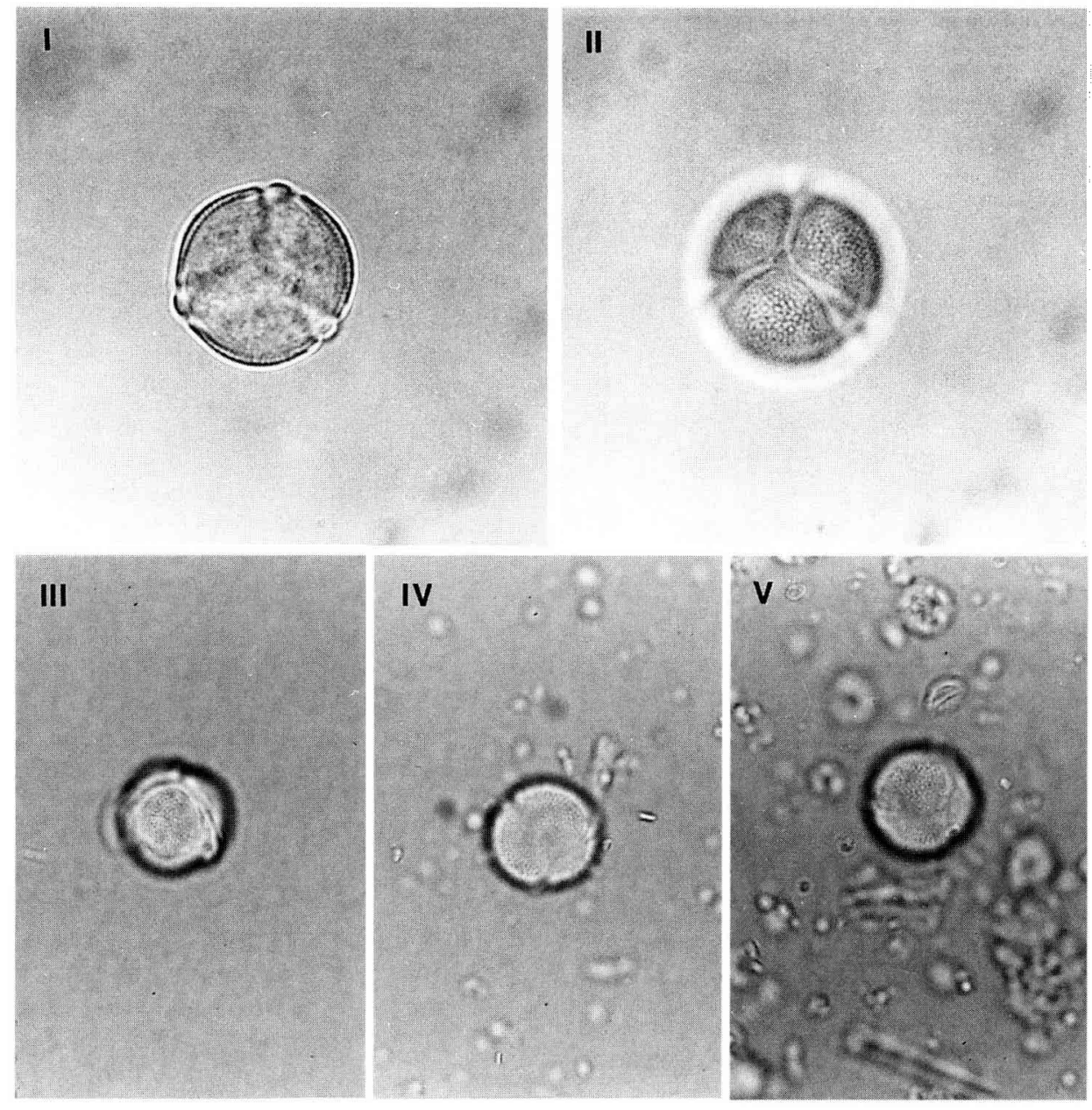

Abb 2. LM-Aufnahmen: Pollen von Aeschynomene americana in Äquatorial-Ebene (I), in Pol-Ebene (II), $\times 1250$ - sowie im Vergleich (III) mit der Cuba-Form aus Mexico (IV)- und Cuba (V)-Honig, $\times 1000$.

Verbreitung von Trockensavannen (Knapp, 1965).

Als Trachtpflanze für Bienen wird $A e$ americana für Venezuela aufgeführt - nachgewiesen in Mérida im Norden des Landes (Lopez-Palacios, 1986), wobei hier neben einheimischen auch eingeführte Pflanzen angegeben sind. Für das tropische Amerika wird $A e$ americana als Pollen- und Nektarspender angegeben, wobei nach Ordetx Ros (1952) die Nektarsekretion hier nicht besonders ergiebig sein soll. Als Provenienzen sind die Antillen, Mexico, Mittelamerika, Gebiete im nördlichen Südamerika sowie Afrika erwähnt. Fichtl und Adi (1994) beschreiben in ihrem Buch sowohl Ae abyssinica als auch Ae americana als gute Bienenweidepflanzen sowohl für Pollen als auch für Nektar, wobei jedoch nur Aeschynomene americana als heimisch im tropischen und subtropischen Amerika angegeben ist. Das verstreut, bis zu $60 \mathrm{~cm}$ hoch 

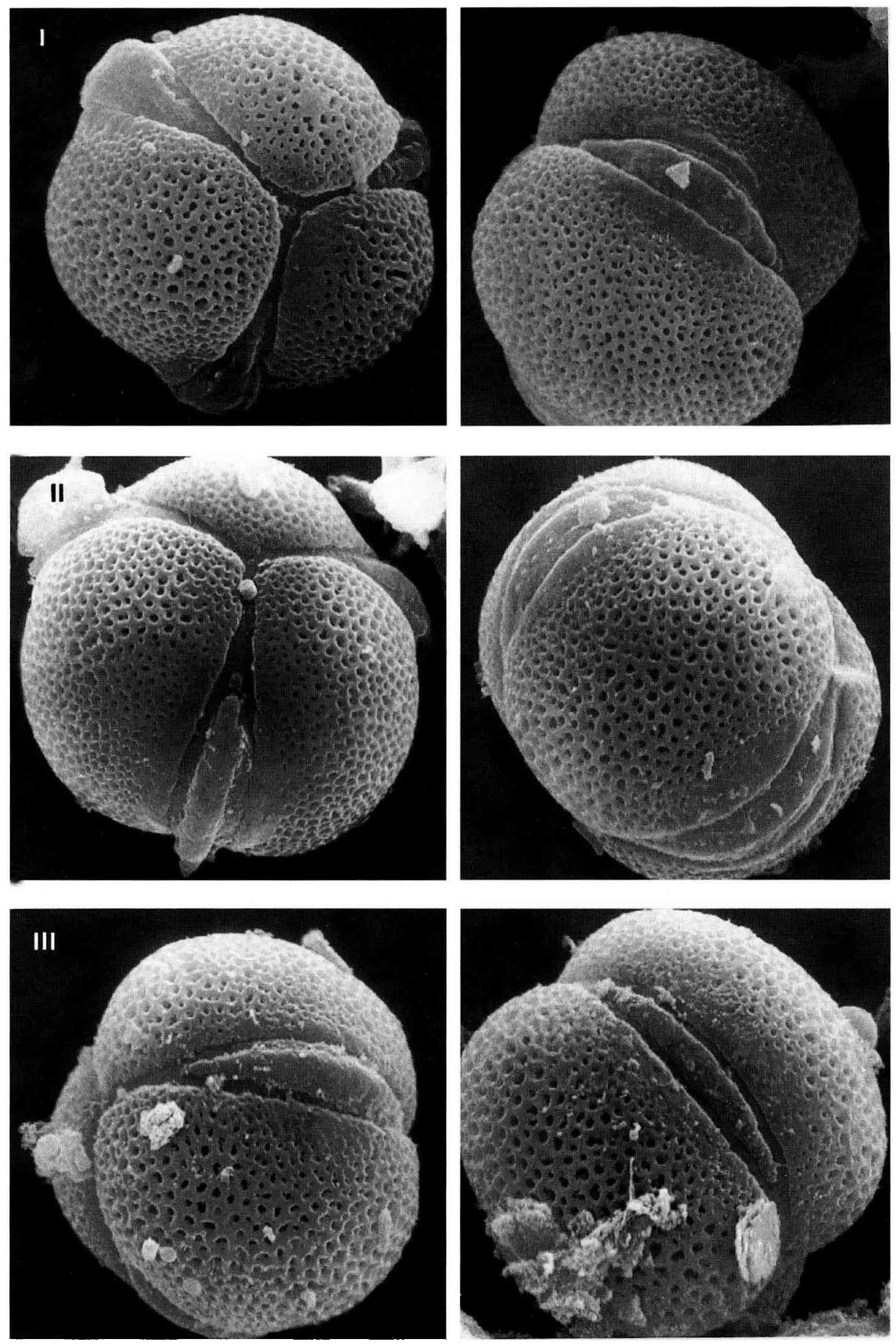

Abb 3. REM-Aufnahmen: Pollen von Aeschynomene americana (I), Cuba-Form aus Mexico (II)- und Cuba (III)-Honig, x 5000 . 
wachsende Kraut wurde in Äthiopien eingebürgert. Man findet es an Straßenrändern, auf gerodeten Waldflächen und sogar kultiviert als Futterpflanze. Fichtl und Adi geben ferner an, daß die Blüten frequentiert von Bienen besucht werden. Bei einem gehäuften Auftreten soll die Pflanze bedeutend für eine Nektartracht sein.

\section{SCHLUSSFOLGERUNG}

Zusammenfassend muß gesagt werden, daß unter Berücksichtigung der Klima- und Vegetationsverhältnisse bei der Verbreitung der unterschiedlichen Aeschynomene-Arten nicht nur aufgrund der Pollenmorphologie sondern auch der gleichartigen Lebensräume die Zugehörigkeit des Pollens zu der Gattung Aeschynomene bestätigt wird. Die oben aufgeführten Beschreibungen zu Herkunft, Häufigkeit und Bedeutung als Bienen-Trachtpflanze der Art Ae americana bestätigen die eingangs erwähnten Vermutungen über den Trachtwert und vervollständigen das Ergebnis der pollenmorphologischen Untersuchungen, daß es sich bei der sog. 'Cuba-Form' um den Pollen von Aeschynomene americana handelt.

\section{DANKSAGUNG}

Wir möchten an dieser Stelle Herrn Reinhard Fichtl für die Überlassung der äthiopischen Pollensammlung sowie Herrn Winfried Henrich für seine Hilfsbereitschaft und die zur Verfügung gestellten Präparate und Honige unseren besonderen Dank aussprechen.

\section{Summary - Identification of a previously unassigned pollen type in Central Ame- rican honeys ('Cuba-form'): Aeschynomene americana L (Legumi- nosae, Papilionoideae). In honeys from Central American provenances such as Mexico (Yucatan, Chiapas) or Guatemala, a}

pollen type ('Cuba-form') occurs as a reliable marker for those regions (fig 1). The species origin for this pollen is unknown to melissopalynologists. Because the 'Cubaform' amounts to up to $16 \%$ of pollen grains in honey, there is an urgent interest to solve this secret. This is very important for the valuation of botanically characterized honey by the criterion of pollen analysis. During palynological work on a collection of Ethiopian pollen grains the 'Cuba-form' was detected. Pollen grains from plant material of Aeschynomene americana and 'Cubaforms' of authentic Mexican and Cuban honeys were prepared for light- and scanningelectron-microscopical analyses (figs 2 and 3 ). The following characterizations were made for all three pollen types: shape: intersemiangular to circular, 3-colporate, $P 16$ $\mathrm{mm}(15-17 \mu \mathrm{m}) \times \mathrm{E} 18.5 \mu \mathrm{m}(17-20 \mu \mathrm{m})$; apertures: syncolporate, ora lolongate, colpi with operculum, margo psilate in equatorial area; exine: sexine reticulate, heterobrochate, lumina up to $0.5 \mu \mathrm{m}$, muri simplicolumellate; colour: colourless, grey. The clear correspondence in morphology indicates that the 'Cuba-form' is the pollen of $A$ americana. Further species of this plant occur in similar climatic regions such as in South Africa ( $A$ elaphroxylon, A nyassana, with little deviations in pollen morphology), Australia and India ( $A$ indica). $A$ abyssinica and $A$ americana are both described as good plants for honey flow in Ethiopia, but only $A$ americana is native in tropical and subtropical America. Pollen morphological, geographical and climatical facts confirm the results.

Résumé - Identification d'un type pollinique non assigné (dit "forme de Cuba») présent dans les miels d'Amérique centrale : Aeschynomene americana $L$ (Leguminosae, Papilionoideae). Un type pollinique dit «forme de Cuba» se rencontre exclusivement dans les miels provenant d'Amérique centrale, par exemple du 
Mexique (Yucatan, Chiapas) ou du Guatémala, pour lesquels il constitue un marqueur fiable (fig 1). Jusqu'à présent l'origine botanique de ce pollen était inconnue des mélissopalynologistes. Parce que la forme de Cuba représente jusqu'à $16 \%$ des grains de pollen dans le miel, il est urgent d'en résoudre l'énigme. Ceci est particulièrement important dans le cas de l'évaluation d'un miel caractérisé botaniquement au moyen de l'analyse pollinique. Au cours d'un travail de palynologie sur une collection de grains de pollen d'Éthiopie, la forme de Cuba a été rencontrée. Des grains de pollen de la plante Ae americana et des formes de Cuba provenant de miels authentiques du Mexique et de Cuba ont été préparés pour être analysés en microscopie optique et à balayage (figs 2 et 3 ). Pour les trois types polliniques les caractères morphologiques sont les suivants : Forme : intersemiangulaire à circulaire, tricolporée, P $16 \mu \mathrm{m}$ (15-17) x E 18,5 $\mu \mathrm{m}$ (17-20). Ouvertures : syncolporées, pores allongés, sillons avec un opercule, zone équatoriale à aspect lisse. Exine : réticulée, hétérobrochée, lumière jusqu'à $0,5 \mu \mathrm{m}$, murs à columelles simples. Couleur : incolore, gris. La correspondance manifeste des caractères morphologiques indique que la forme de Cuba est un fait un pollen d'Ae americana. D'autres espèces de cette plante sont présentes dans des régions climatiques similaires, en Afrique du Sud (Ae elaphroxylon, Ae nyassana - avec de petites différences dans la morphologie polinique), en Australie et en Inde (Ae indica). Ae abyssinica et $A e$ americana sont toutes deux décrites comme des plantes fournissant de bonnes miellées en Éthiopie, mais seule Ae ame- ricana est indigène en Amérique tropicale et sub-tropicale. Les données climatiques et géographiques confirment les résultats de la morphologie pollinique.

\section{mélissopalynologie / miel / Amérique centrale / "forme de Cuba"}

\section{REFERENCES}

Beadle NCW (1981) The Vegetation of Australia. Gustav Fischer Verlag, Stuttgart

Beard JS (1990) Plant Life of Western Australia. Kangaroo Press, Kenthurst, NSW

Erdtman G (1952) Pollen Morphology and Plant Taxonomy - Angiosperms. Almquist \& Wiksells, Uppsala

Faegri K (1993) Bestimmungsschlüssel für die nordwesteuropäische Pollenflora. Gustav Fischer Verlag, Stuttgart

Fichtl R, Adi A (1994) Honeybee fiora of Ethiopia. DED, Margraf Verlag, Weikersheim

Knapp R (1965) Die Vegetation von Nord- und Mittelamerika. Gustav Fischer Verlag, Stuttgart

Louveaux J et al (1978) Methods of Melissopalynologie. Bee World 59, 139-157

Lopez-Palacios S (1986) Catalogo para una Flora Apicola Venezolana. Talleres Gráficos Universitarios, Mérida, Venezuela

von der Ohe K, Dustmann JH (1990) Raster-elektronenmikroskopische Studien an Pollen aus Bienenhonig. III. Der Harmomegathie-Mechanismus und seine Auswirkungen auf die Exine-Strukturen am Beispiel unterschiedlicher Pollentypen. Apidologie 21, 293-302

Ordetx Ros GS (1952) Flora Apicola de la America Tropical. Editorial Lex, La Habana, Cuba

Willis JS (1973) A Dictionary of the Flowering Plants and Ferns. University Press, Cambridge

Zander R (1993) Handwörterbuch der Pflanzennamen. Auflage 14, Ulmer Verlag, Stuttgart

van Zinderen Bakker EM (1959) South African Pollen Grains and Spores, Part III. A A Balkema, Amsterdam/Cape Town 